\title{
RIGHLIGHTS
}

PROSTATE CANCER

\section{Novel technique for early detection of lymph node metastases}

Metastasis to regional lymph nodes is an important prognostic factor in prostate cancer. Disease progression and patient survival can vary depending on the number of metastatic lymph nodes identified and the tumor volume. Histopathological analysis of a few sections cut from each lymph node is the current standard assessment technique. Malignant cells are, however, often unevenly distributed throughout the node. As such, histological analysis does not always generate an accurate report of tumor spread or load.

David Schilling and colleagues report a novel technique for the early detection of tumor cell dissemination, and estimation of tumor cell burden, in the lymph nodes of men with prostate cancer. They based their method on a technique originally used by another research group to detect lymph node metastases in patients with melanoma.

Schilling et al. analyzed 232 lymph nodes (an average of 22 per patient) from
20 men with prostate cancer (median age 66 years) who were at high risk of lymph node involvement. Each node was bisected. One half was examined using routine histopathology and step-section analysis. The other half was mechanically disaggregated before being processed for single-cell immunocytochemistry to detect pancytokeratine. Ten pelvic lymph nodes from two male patients not suspected of having prostate cancer were used as controls.

Metastases were detected in 8 of 20 patients using standard histopathology, and in 14 of 20 patients using singlecell analysis. Routine histology revealed invasion of 25 of the 232 lymph nodes tested, the single-cell technique more than twice that, at 52 nodes.

Immunocytochemical detection of cancerous cells in lymph node tissue that was negative according to histopathological analysis led the investigators to conclude that the former technique is more sensitive. Automation of the single-cell

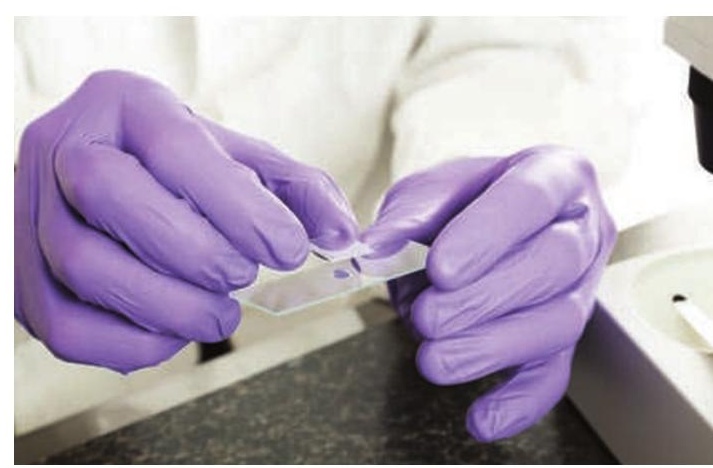

approach could make it a useful adjunct to the standard histological workup in men with high-risk prostate cancer. Whether the increased likelihood of detecting micrometastases translates into clinically important improvements in prognostication remains to be seen.

\section{Rebecca Drake}

Original article Schilling, D. et al. Quantification of tumor cell burden by analysis of single cell lymph node disaggregates in metastatic prostate cancer. Prostate 70, 1110-1118 (2010) 